\title{
Constitutive Overexpression of Bcl-2, Survivin and ER Stress Chaperone GRP-78 Confers Intrinsic Radioresistance in Human Hepatocellular Carcinoma Cells: Insight into the Mechanistic Pathways Involved
}

\author{
Chih-Hsiung Wu ${ }^{1 \#}$, Yih-Huei Uen ${ }^{2,3}$, Chun-Te Ho ${ }^{4}$, Ya-Ting Tseng ${ }^{5}$, Tsan-Zon Liu ${ }^{6}$, \\ Jeng-Fong Chiou ${ }^{6 \dagger}$, Stephen Wan Leung ${ }^{7 \dagger}$ \\ ${ }^{1}$ Department of Surgery, Taipei Medical University-Shuang Ho Hospital, Taipei, Chinese Taipei; ${ }^{2}$ Department of General Surgery, \\ Chi-Mei Foundation Medical Center, Tainan, Chinese Taipei; ${ }^{3}$ Departmento of Electrical Engineering, South Taiwan University, \\ Tainan, Chinese Taipei; ${ }^{4}$ Graduate Institute of Medical Sciences, Taipei Medical University, Taipei, Chinese Taipei; ${ }^{5}$ Graduate Insti- \\ tute of Medical Biotechnology, Chang-Gung University, Gueishan, Chinese Taipei; ${ }^{6}$ Cancer Center and Department of Radiation \\ Oncology, Taipei Medical University and Hospital, Taipei, Chinese Taipei; ${ }^{7}$ Department of Radiology and Nuclear Medicine, Yuan's \\ General Hospital, Kaohsiung, Chinese Taipei. \\ Email: ${ }^{\dagger}$ sjfchiou@ms68.hinet.net, ${ }^{\dagger}$ lawn@ms36.hinet.net
}

Received December $18^{\text {th }}, 2012$; revised January $20^{\text {th }}, 2013$; accepted January $29^{\text {th }}, 2013$

\begin{abstract}
We present evidence here that abundantly expressed $\beta$-catenin-triggered NF- $\kappa$ B-dependent upregulation of inducible nitric oxide synthase (iNOS) found in hepatoma Mahlavu cells (RT-resistant variant designated as RR-Mal), but not in Hep 3B cells (RT-sensitive variant designated as RS-3B) is a key element contribrting to the radioresisitance through the activation of two prominent radioprotective pathways. First, high iNOS expression found in RR-Mal, but not in RS-3B cells was found to perturb calcium homeostasis that triggered ER stress response leading to the overproduction of ER chaperone GRP-78 via robust generation of cleaved ATF- $6 \alpha(50 \mathrm{kDa})$ subunits and their nuclear translocation. Meanwhile, both abundantly expressed NF- $\kappa \mathrm{B}$ and COX-2 found in RR-Mal cells could also provoke an increased production of PGE2 resulting in robust production of Bcl-2. Interestingly, when RR-Mal cells were treated with PDTC (a NF- $\kappa$ B inhibitor) or celecoxib (a COX-2 inhibitor), a concentration-dependent downregulation of Bcl-2 could be demonstrated implying that Bcl-2 overexpression was indeed mediated through $\mathrm{NF}-\kappa \mathrm{B} / \mathrm{Cox}-2 / \mathrm{PGE} 2$ pathway. Importantly, we also unveiled that siRNA-mediated silencing of survivin in RR-Mal cells could result in a concomitant downregulation of GRP-78 due to a severe inhibition of ATF- $6 \alpha(50 \mathrm{kDa})$ expression. Taken together, our data demonstrate that constitutively overexpressed $\beta$-catenin/NF- $\kappa \mathrm{B} / \mathrm{iNOS}$ and NF- $\kappa \mathrm{B} / \mathrm{COX}-2 / \mathrm{PGE} 2$ pathways that overproducing GRP-78, survivin and Bcl-2 expressions are responsible for radioresistance acquisition in RR-Mal cells. Thus, both pathways could be served as potential targets for overcoming radioresistance.
\end{abstract}

Keywords: Radioresistance; Hepatoma Cells; Inducible Nitric Oxide Synthase; Bcl-2; Survivin; GRP-78

\section{Introduction}

Hepatocellular carcinoma (HCC) is one of the most common visceral neoplasms worldwide and one of the rare human neoplasms etiologically linked to viral factors. Chronic infection with the hepatitis B virus (HBV) and the hepatitis $\mathrm{C}$ virus (HCV) have implicated in about $80 \%$ of case worldwide [1]. The annual incidence of HCC in the United States has risen sharply, tripling in the past 20 years, while the overall incidence of cancer has

\footnotetext{
*We declare no competing interests.

${ }^{\#}$ Both authors contribute equally.

${ }^{\dagger}$ Corresponding authors.
}

stabilized $[2,3]$. The trend in HCC incidence will certainly continue because of the large pool of people chronically infected with $\mathrm{HCV}$. HCV is a particularly lethal cancer: more than $95 \%$ of HCC patients die as a result of disease progression. In Taiwan, $\mathrm{HCC}$ is also one of the leading causes of cancer-related death among men, and is the second among women [4]. Therefore, HCC will conceivably remain a major public health problem for sometime to come.

Surgical resection has been the major treatment modality for HCC, but owing to the lack of early detection markers, HCC is often diagnosed at an advanced stage. 
For this reason, only a small fraction of patients can qualify for surgery. Chemotherapy remains the major treatment option for HCC, but despite the use of diverse chemotherapeutic compounds and treatment modalities, the survival rate has not improved for more than two decades. Multifaceted mechanisms are probably responsible for the low response rates, primary of which is intrinsic drug resistance [5].

Ionizing radiation (IR) is a widely used therapy for solid tumor and about $50 \%$ of cancer patients in the United States receive radiation therapy [6]. Radiotherapy (RT) is a highly effective treatment for cancer. The targets of RT are both tumor cells and tumor vasculature. IR is thought to act by direct targeting tumor clonogens, also known as stem cells. Tumor curability is believed to be determined by the most resistant clonogens because one surviving stem cell appears sufficient for reconstituting tumor growth. Tumor response to RT was found to be regulated by endothelial cell apoptosis. RT is often used to treat HCC patients when portal vein thrombosis occurred or tumor that was too close to major blood vessels to have good transarterial embolization effect. Along the same vein, the administration of chemotherapy either alone or in combination with RT is an important factor in reducing the mortality and morbidity of cancer patients. Resistance to both chemotherapy and radiotherapy represents a major obstacle to a successful outcome. The identification of novel biomarkers or pathways that can be used to predict treatment response is of vitally importance in overcoming intrinsic radioresistance and would allow therapy to be tailored on an individual patient basis.

The major focus of the current study is to identify the genetically endowed radioresistance-associated molecular signatures of an established HCC clonal variant and to shed some lights into the mechanistic pathways that are involved in regulating the expression of these radioresistance effctors.

\section{Methods and Materials}

\subsection{Cell Culture}

Two HCC cell lines, Hep3B and Mahlavu used in this study were grown in medium consisting of Dulbecco's modified Eagle's medium (Life Technologies, NY) supplemented with $10 \%(\mathrm{v} / \mathrm{v})$ fetal bovine serum in $5 \% \mathrm{CO}_{2}$, $37^{\circ} \mathrm{C}$ humidified incubator.

\subsection{Irradiation Technique}

The hepatoma cells (Mahlavu and Hep3B) were subcultured in glass cover slip and the cells were allowed to grow for 3 days before irradiation. The culture media was replaced with HESS buffer before the cells were treated with a linear accelerator (Varian Medical System, Palo Alto, CA, USA) using 6 megavolts of energy with a source-to-target distance of $100 \mathrm{~cm}$. The cells were placed on a $1-\mathrm{cm}$ bolus and treated with a posterior anterior direction portal to allow a $1-\mathrm{cm}$ radiation buildup. A radiation absorption dose of $16 \mathrm{~Gy}$ in a single fraction was delivered to the hepatoma cells.

\subsection{TUNEL Assay}

The treated cells were then fixed with 3.7\% paraformaldehyde in medium 6 hrs after irradiation. DNA fragmentation was detected by terminal deoxynucleotidly transferase-mediated nick end labeling (TUNEL) using an Apo-BrdU in situ DNA Fragmentation Assay Kit (BioVision, Mountain View, CA) according to vendor's instructions. Cells were stained for apoptosis using a confocal microscope to determine the percentage of the cells that have undergone programmed cell death.

\subsection{Confocal Microscopic Imaging}

To measure the production of intracellular NO, HCC cells were cultured in poly-L-lysine coated slides in $3-\mathrm{cm}$ culture dishes. After reaching $80 \%$ cell density, cells were incubated with $2 \mu \mathrm{M}$ DAF-FM diacetate in HEPES for $30 \mathrm{~min}$ in the dark and imaged using a Leica SP2 laser scanning confocal microscope (Leica-Microsystems, Mannheim, Germany). To measure cytosolic calcium concentrations, the same set of HCC cells were stained with $2 \mu \mathrm{mM}$ Fluo-4 to detect mitochondrial and cytosolic calcium, respectively. Fluorescent images of Fluo-4 were obtained. At the end of each experimental period, cells were then mounted on the stage of a Leica SP2 laser scanning confocal microscope. The cells were randomly selected and imaged.

\subsection{Enzyme-Linked Immunoassay (ELISA)}

Endogenous Bcl-2 expression in Mahlavu and Hep3B cells was measured using an ELISA kit (Endogen, Woburn, MA). Briefly, 96-well microplates were precoated with a mouse monoclonal antihuman Bcl-2 antibody, and samples $(50 \mathrm{~L})$ were diluted 1:1 (v/v) with fluorescein isothiocyanate (FITC)-labeled secondary antibody in triplicate. The plates were incubated for $2 \mathrm{~h}$ at room temperature, washed, and followed by horseradish peroxide-labeled anti-FITC antibody. After 30 min incubation, plates were washed, and TMB/peroxide was added for color development. The reaction was stopped with sulfuric acid, and the optical density was measured at $450 \mathrm{~nm}$ using a microplate reader (Bio-Rad Laboratories, Philadelphia, PA). A Bcl-2 standard curve was generated to quantitate the amount of $\mathrm{Bcl}-2$ in Units/mg total protein. One unit is defined as the amount of $\mathrm{Bcl}-2$ protein 
in 1000 lysed cells of an internal control cell line (HL60 ). Intra-assay coefficients of variance were less than $8 \%$ in all measurements.

Endogenous $\mathrm{PGE}_{2}$ expression in Mahlavu and Hep3B cells was measured using an ELISA EIA kit (Cayman Chemical Company, MI, USA). Mahlavu and Hep-3B were seeded $1 \times 10^{6}$ in Dulbecco's Modified Eagle Medium and $10 \mathrm{~cm}$ culture dish at $37^{\circ} \mathrm{C}, 5 \% \mathrm{CO}_{2}$ and incubated for $80 \%$ confluence. We collected and washed cells 2 - 3 time by 1x PBS solution. We then lysed cell by RIPA Lysis Buffer System (Santa Cruz, Inc.), and the cell lysates were incubated with enzyme immunoassay buffer (contained $50 \mathrm{mM}$ Tri-HCl (ph 7.4), $100 \mathrm{mM}$ $\mathrm{NaCl}, 1 \mathrm{mM} \mathrm{CaCl} 2,1 \mathrm{mg} / \mathrm{ml} \mathrm{D}$-glucose, $28 \mathrm{M}$ indomethacin) in ice for $30 \mathrm{sec}$. And centrifuge sample at $1000 \mathrm{~g}, 4^{\circ} \mathrm{C}$ for 15 mins and collecting supernatants for the detection of $\mathrm{PGE}_{2}$ according to vendor's instructions.

\subsection{Western Blot Analysis}

Cells plated at $1 \times 10^{6}$ per 10 -cm culture dishes were allowed to grow for $24 \mathrm{hrs}$ at $37^{\circ} \mathrm{C}$ in a $5 \% \mathrm{CO}_{2}$ incubator and then treated with various concentrations of PDTC $(25$ to $50 \mathrm{~g} / \mathrm{ml}$ ) and celecoxib $(25$ to $75 \mathrm{~g} / \mathrm{ml}$ ) for $24 \mathrm{hrs}$. Protein samples were collected in RIPA buffer $(50 \mathrm{mM}$ Tris. $\mathrm{HCl} \mathrm{pH} \mathrm{7.5,} 150 \mathrm{mM}$ sodium chloride, $1 \mathrm{mM}$ EDTA, $1 \%$ Triton X-100, $0.25 \%$ sodium deoxycholate, $0.1 \%$ SDS ) containing $50 \mathrm{mM}$ sodium fluoride, $1 \mathrm{mM}$ sodium orthovanadate, $10 \mathrm{mM}$ b-glycerolphosphate and 1x protease inhibitor Cocktail (Sigma-Aldrich, St Louis, MO). Protein concentrations were assayed using Bio-Rad protein Assay Kit (Bio-Rad, Richmond, CA). Protein extracts representing $40 \mu \mathrm{g}$ were separated on a $12 \%$ SDS-PAGE gel and electrophoretically transferred to a polyvinylidene difluoride (PVDF) membrane. Membranes were blocked in 5\% skim milk powder in TBST solution (Tris-buffered saline containing $0.1 \%$ Tween 20 ) for $1 \mathrm{hr}$ at room temperature followed by incubation with first antibodies against COX-2 (1:500), Grp78 (1:1000), ATF-6 (50 kDa) (1:1000), Bcl-2 (1:1000), Survivin (1:1000) [Sata Cruz Biotech, Santa Cruz, CA]; $\beta$-catenin $(0.3 \mathrm{~g} / \mathrm{ml})$ [Upstate], phosphate-Akt (1:4000) [Epitomics], NFkB (1:5000) [Millipore], iNOS (1:250) [BD Biosciences] and actin $(1: 10,000)$ [Sigma-Aldrich, St. Louis, $\mathrm{MO}$. Following washing with TBST solution, blots were incubated with the appropriate HRP-labelled secondary antibody for $1 \mathrm{hr}$ at room temperature. The antigen-antibody complexes were detected by the enhanced chemiluminescence $(\mathrm{ECL})$ with a chemiluminescence analyzer.

\subsection{Nuclear Protein Extraction}

Cell pellet added $200 \mu \mathrm{l}$ Buffer A [10 mM HEPES (pH 7.9) (Sigma), $10 \mathrm{mM} \mathrm{KCl} \mathrm{(Sigma),} 0.1 \mathrm{mM}$ EDTA, 0.1 mM EGTA (Sigma), $1 \mathrm{mM}$ DTT, $0.5 \mathrm{mM}$ PMSF
(Sigma)], mixed gently, and added $6.25 \mu 1$ 10\% NP-40 (Fluka) in ice for 20 minutes (mixed gently every $5 \mathrm{~min}$ utes). Then, we used centrifuge at $12,000 \mathrm{rpm}$ in $4^{\circ} \mathrm{C}$ for $30 \mathrm{sec}$ and collected supernatants (cytosol fraction) in new Eppendorf. The remined pellets added $50 \mu \mathrm{l}$ Buffer B [20 mM HEPES (pH 7.9), $0.4 \mathrm{M} \mathrm{NaCl}$ (Sigma), $1 \mathrm{mM}$ EDTA, 1 mM EGTA, $1 \mathrm{mM}$ DTT, $1 \mathrm{mM} \mathrm{PMSF}, 2 \mathrm{~g} / \mathrm{ml}$ leupeptin (Sigma), $2 \mathrm{~g} / \mathrm{ml}$ aprotinin (Sigma), 1\% protease inhibitor cocktail (Sigma)] vortex strongly for $15 \mathrm{sec}$ in ice for 10 minutes and repeated vortex strongly. Then we used centrifuge at $12,000 \mathrm{rpm}$ in $4^{\circ} \mathrm{C}$ for 20 minutes and collected supernatants (nucleus fraction) in new eppendorf. Then the samples were detected by Western blot.

\section{8. siRNA Interfering Technique}

The expression of GRP-78 was ablated in Mahlavu cells with small interfering RNA (siRNA). Iubrief, the target sequence for the human GRP-78 mRNA was 5'- AAGGTTACCCATGCAGTTGTT - 3 '. The scrambled siRNA sequence was 5'-AAGGTGGTTGTTTTGTTCACT-3'. The GRP-78 and scramble siRNA were inserted into pSUPERIOR vector and transfected into Mahlavu cells and selected the stably transfected cells by antibiotic as previous described [7]. Futher, surviving expression in Mahlavu cells was also ablated using the same procedure as previously described by us [8]

\subsection{Statistical Analysis}

All data was presented as means \pm standard derviation from at least 3 independent experiments and analyzed using Student's $t$ test. A $p$ value of less than 0.05 was considered statistically significant.

\section{Results}

\subsection{Constitutively Overexpressed Bcl-2, Survivin and GRP-78 Confers Intrinsic Radioresistance}

The complexicity of radioresistance acquisition makes it necessary to identify a panel of radioresistance-related effectors rather than a single determinant that may be involved in a radioresistant cancer cell. For this reason, we set out to investigate what may be the possible effectors associated with the radioresistance acquisition of HCC Mahlavu cells (Figure 1). Our data clearly demonstrated that simultaneously overexpressed Bcl-2, survivin and ER stress chaperone GRP-78 compoundedly contributed to the intrinsic radioresistance of Mahlavu cells. These data underscore the important of a notion that using a single determinatnt rather than a composite profile of radioresistant effectors is insufficient to pinpoint the mechanism associated with radioresistance of cancer 
cells. Therefore, to identify the expression of multiple radioresistant effectors and their related mechanistic pathways are utmost important because they can provide the basis for designing appropriate strategies for overcoming radioresistance in order to improve the efficacy of radiotherapy.

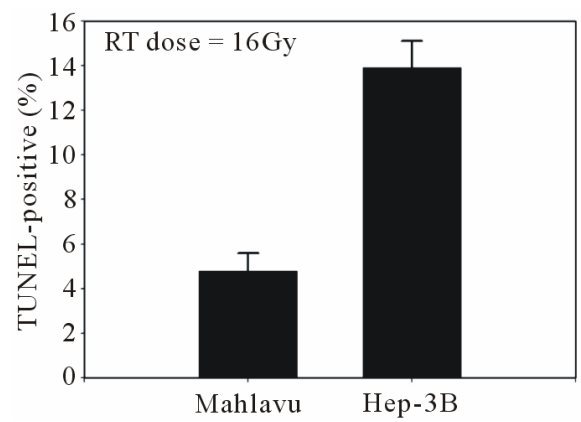

Figure 1. Comparative radioresistance in two p53-mutant of HCC cells. When both HCC cells were irradiated with a single dose of $16 \mathrm{~Gy}$, Mahlavu subline was shown to be remarkably more radioresistant that its pair-matched Hep3B subline as judged by the \% of TUNEL-positive fractions existed between the two HCC sublines being tested.

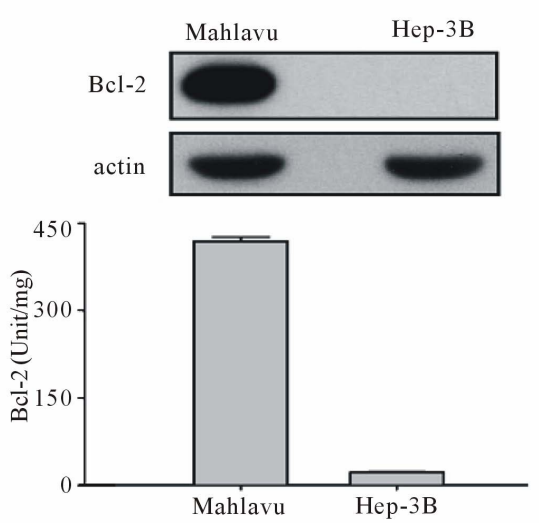

(a)

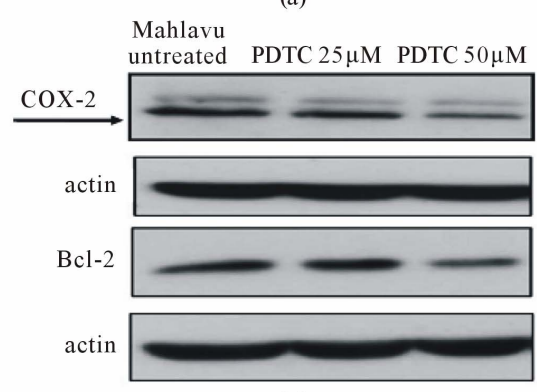

(c)

\subsection{Evidence That Radioresistance Associated with Constitutive Overexpression of Bcl-2 Is Mediated through NF- $\mathrm{kB} / \mathrm{COX}-2 / \mathrm{PGE}_{2}$ Pathway}

As revealed in Figure 2, radioresistant Mahlavu cells constitutively overexpressed Bcl-2 was intimatedly associated with plethoric expressions of $\mathrm{COX}-2$ and $\mathrm{PGE}_{2}$. These data clearly indicated that $\mathrm{Bcl}-2$ production was derived through NF- $\kappa \mathrm{B} / \mathrm{COX}-2 / \mathrm{PGE}_{2}$ Pathway. To further substantiate this notion, we used PDTC (a NF- $\kappa$ B inhibitor) and celecoxib (a COX-2 inhibitor) to treat Mahlavu cells and found out that both compounds caused a concentration-dependent downregulation of Bcl-2. These data further substantiate that $\mathrm{Bcl}-2$ overexpression was indeed mediated through NF- $\kappa \mathrm{B} / \mathrm{COX}-2 / \mathrm{PGE}_{2}$ Pathway.

\subsection{Evidence That Radioresistance Associated with Constitutive Overexpression of Survivin Is Mediated through $\beta$-Catenin/NF- $\alpha$ B/iNOS Pathway}

As also revealed in Figure 3, radioresistant Mahlavu cells constitutively overexpressed survivin was also as-

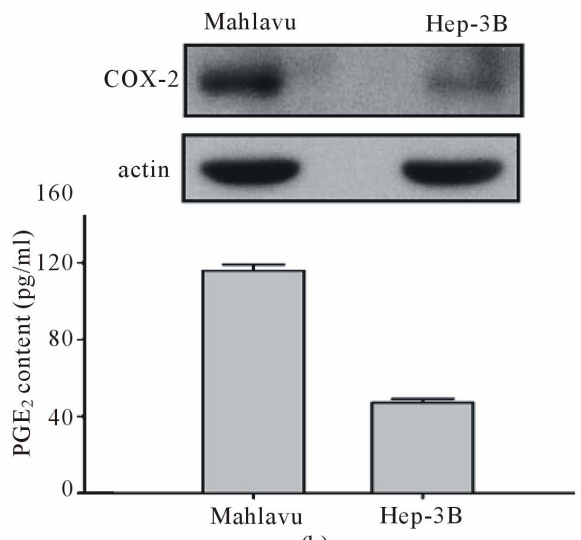

(b)

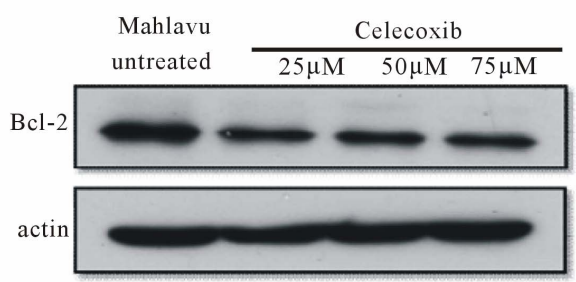

(d)

Figure 2. Comparative assessment of the expression of Bcl-2, a major pro-surrvival factor and insight into the mechanistic pathway involved. (a) Quantitatively, the endogenous Bcl-2 level in Mahlavu cells was approximately 20-fold higher than Hep 3B cells (419 \pm 7 units/mg and $22 \pm 1$ units/mg, respectively); (b) Constitutively overexpressed COX-2 was found in Mahlavu, but not in Hep3B cells as detected by Western blot. Quantitatively, the endogenous PGE 2 production of Mahlavu cells was approximately 2.5-folc higher than Hep 3B cells $(110 \pm 8 \mathrm{pg} / \mathrm{ml}$ and $45 \pm 4 \mathrm{pg} / \mathrm{ml}$, respectively); (c) Treatment of Mahlavu cells with PDTC (a NF- $\kappa$ B inhibitor) resulted in a dose-dependent inhibition of cox-2 and Bcl-2 expression; (d) Treatment of Mahlavu cells with celecoxib (a cox-2 inhibitor) resulted in inhibition of Bcl-2 expression. 


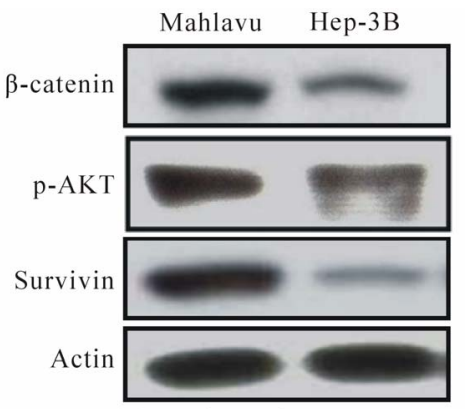

(a) Total protein extract

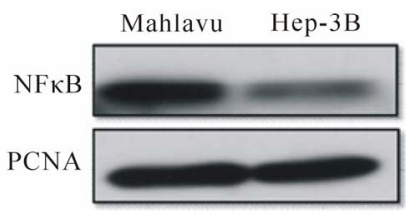

(b) Nuclear protein extract

Figure 3. Comparative assessment of the expression of survivin, a radioprotective effector and insight into the mechanistic pathway involved. (a) Constitutively overexpressed survivin, $\beta$-catenin and P-Akt were found to be associated with Mahlavu, but not in Hep 3B cells as detected by Western blot blot; (b) Comparatively, nuclear translocation of NF- $\boldsymbol{K}$ B was found to be abundantly existed in Mahlavu, but not in Hep $3 \mathrm{~B}$ cells implying the involvement of $\beta$-catenin/ $\mathrm{NF}-\boldsymbol{\kappa} \mathrm{B} / \mathrm{p}$-Akt pathway in robust expression of survivin.

sociated with the overexpressions of $\beta$-catenin, phosphorylated Akt, iNOS and nuclear NF- $\kappa \mathrm{B}$. These data implicated that $\beta$-catenin/NF- $\kappa \mathrm{B} / \mathrm{iNOS}$ Pathway might be involved. Interestingly, siRNA silencing of survivin on Mahlavu cells caused concomitantly the downregulation of $\beta$-catenin implying that survivin expression was modulated by $\beta$-catenin through Wnt signaling pathway for which survivin and iNOS were the downstream genes regulated by this pathway.

\subsection{Evidence That Radioresistance Associated with Constitutive Overexpression of ER Chaperone GRP-78 Is Mediated through ER Stress Response via Activating the Cleavage of ATF-6 $\alpha$}

As revealed in Figure 4, radioresistant Mahlave cells constitutively overexpressed GRP-78 was associated with the activation of ER stress response resulting from a sustained nitric oxide (NO) generation via higher iNOS that was genetically endowed. Consequently, cytosolic calcium $\left(\mathrm{cCa}^{2+}\right)$ homeostasis was perturbed leading to the occurrence of ER stress response that initiated the cleavage of ATF- $6 \alpha(90 \mathrm{kDa})$ transcription factor. The cleaved ATF-6 $\alpha(50 \mathrm{kDa})$ then translocated to the nuclear region to cause releasing of GRP-78. Interestingly, siRNA of silencing of survivin completely blocked the formation of ATF- $6 \alpha(50 \mathrm{kDa})$, leading to the severe downregulation of GRP-78. Even more importantly, siRNA silencing of GRP-78 also downregulated $\beta$-catenin formation implying that the cross-talk between survivin and GRP-78 was in operation (Figure 5).

\section{Discussion}

Statistical data revealed that more than $50 \%$ of patients diagnosed with cancer were treated with radiotherapy

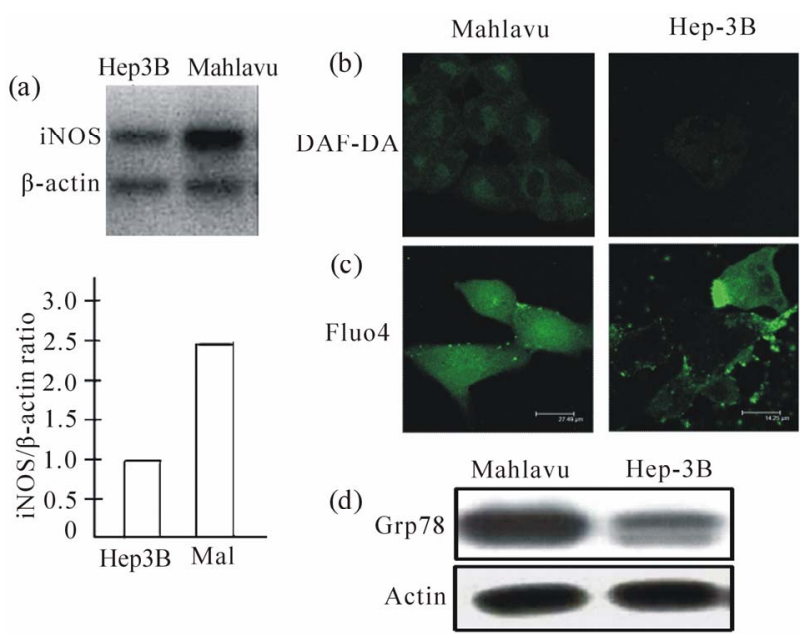

Figure 4. Constitutively overexpressed iNOS and NO production were found to be associated with Mahlavu, but not in Hep 3B cells (a) and (b); (c) Higher NO production observed in Mahlavu cells was found to perturb cytosolic calcium homeostasis which consequently promoted ER stress response resulting in the overexpression of ER chaperone protein GRP78 (d).

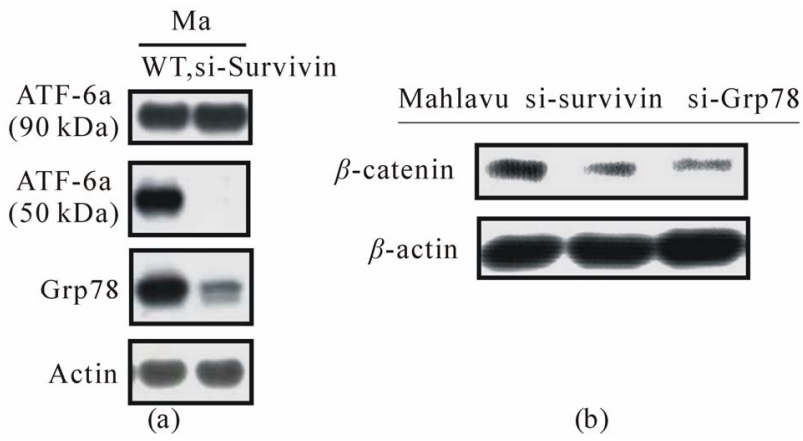

Figure 5. (a) ER stress response-triggered overexpression of ER chaperone protein GRP78 found in Mahlavu cells was shown to be proceeded via the cleavage of ATF-6 $\alpha$ (90 kDa) to generate plethoric ATF-6 $\alpha$ (50 kDa) subunits and their nuclear translocation which caused the release of GRP78. Interestingly, siRNA silencing of survivin resulted in the complete blockade of ATF-6 $\alpha$ (50 kDa) formation which severely suppressed GRP78 expression; (b) Both siRNA silencing of survivin and GFP78 in Mahlavu cells simultaneously caused severe repression of $\beta$-catenin implying that the latter effector was intimately associated with the expression of survivin and GRP78. 
(RT) [6]. RT is often used to treat the patients with hepatocellular carcinoma (HCC) when portal vein thrombosis occurred for tumor that was too close to major blood vessel to have good transarterial embolization effect. However, both intrinsic and acquired radoresistances, continues to be a major and largely unsolved problem in the radiotherapeutic treatment of liver cancer. This fact underscores the importance of understanding the root causes of how cancer cells poise themselves to acquire radioresistant phenotype and/or stimuli-mediated upregulation of prosurvival pathways that forestall apoptosis and become radioresistance-prone. Information of this sort can be of value in the development of effective strategy for overcoming radioresistance acquisition.

Using a pair of p53 non-functional human $\mathrm{HCC}$ as cell models with distinct disparity in radioresistance (Figure 1), we unequivocally unveiled that Mahlavu (a radioresistant subline) can uniquely and simultaneously overexpressed a composite profile of three prominent radioresistant effectors, namely: Bcl-2, survivin and ER chaperone protein GRP-78 when compared to its pairmatched Hep 3B (a radiosensitive subline). Undoubtedly, these radioresistant effectors compoundedly contribute to the radioresistance acquisition of Mahlavu cells. Thus, the goal of the current study is to gain more detailed insight into the pathways that underlying the overexpression of these radioresistant effectors. Knowledge gain from these studies may help in the development of new effective strategies to overcome intrinsic radioresistance in order to improve the efficacy of radiotherapy.

Overexpression of Bcl-2 has been established as a molecular marker of radioresistance in a variety of cancer types through its function of forestalling apoptosis during radiotherapy [9-14]. However, whether there is an unifying mechanism for regulating Bcl-2 overexpression among various cancer types is thus far incompletely understood. Based on this premise, we thus set out to identify the possible mechanistic pathways involved that underlying Bcl-2 overexpression. As analogus to other cancer types, radioresistant HCC Mahlavu cells were estimated to have a significantly higher intrinsic Bcl-2 expression $(419 \pm 7 \mathrm{u} / \mathrm{mg})$ than radiosensitive Hep 3B $(22 \pm 1 \mathrm{u} / \mathrm{mg}$ ) cells did (Figure 2(a)). Further comparative assessment of molecular signatures between both HCC cells revealed that Mahlavu cells significantly upregulated transcription factor nuclear factor kappa $\mathrm{B}$ $(\mathrm{NF}-\kappa \mathrm{B})$ and its downstream gene cyclooxygenase-2 (COX-2) (Figure 2(b)). Furthermore, we also unveiled that Mahlavu cells could express higher levels of prostaglandin E2 (PGE2) $(116 \pm 3 \mathrm{pg} / \mathrm{ml})$ than Hep 3B (48 \pm $2 \mathrm{pg} / \mathrm{ml}$ ) cells did. Collectively, these data implicate that overexpression of $\mathrm{Bcl}-2$ observed in Mahlavu cells is mediated through $\mathrm{NF}-\kappa \mathrm{B} / \mathrm{COX}-2 / \mathrm{PGE} 2$ pathway. Inter- estingly, when Mahlavu cells were treated with pyrrolidine dithiocarbamate (PDTC) (a NF- $\kappa \mathrm{B}$ inhibitor) or celecoxib (a COX-2 inhibitor) [15], a concentration-dependent downregulation of Bcl-2 expression could be demonstrated. These data further support the notion that overexpression of $\mathrm{Bcl}-2$ is indeed mediated through $\mathrm{NF}-\kappa \mathrm{B} / \mathrm{COX}-2 / \mathrm{PGE} 2$ pathway. Our data are in consistent with the findings observed in colorectal cancer cells indicating that NF- $\kappa \mathrm{B}$ activation provides a prosurvival response to radiation by inhibition of apoptosis and induces COX-2. Curcumin, a polyphenol from the rhizomes of Curcumel longa has been demonstrated to block NF- $\kappa$ B-mediated signaling pathway and potentiates the efficacy of radiation therapy [16]. Furthermore, it has also been documented that aspirin can induce apoptosis in esophageal cancer cells by inhibiting the pathway of NF- $\kappa$ B downstream regulation of COX-2 [17]. Based on these informations, it can be inferred that the genetically endowed radioresitance of HCC cells can be partially overcome by blocking constitutively expressed NF- $\kappa \mathrm{B}$ through the usage of curcumin and aspirin. This possibility is currently under investigation in this laboratory.

Survivin, originally identified as a member of the inhibitor-of-apoptosis (IAP) family, is a bifunctional protein that acts as a suppressor of apoptosis and plays a central role in cell division [18]. The protein is strongly expressed in most common cancer, and has prognostic relevance because high survivin expression has been associated with tumor resistance to chemotherapy and radiotherapy $[19,20]$. In analogous to other cancer cell lines, we also found out that radioresistant HCC Mahlavu cells constitutively overexpressed survivin (Figure 3(a)). Therefore, we proceeded to examine the possible mechanistic pathways that provoking the overexpresseion of survivin in HCC Mahlavu cells. In our preliminary examination of a panel of possible radioresistant associated effectors by Western blot, we found out that Mahlavu cells could also uniquely overexpress $\beta$-catenin and phosphorylated Akt (p-Akt) simultaneously besides NF$\kappa \mathrm{B}$ (Figure 3). These data are in agreement with a number of more direct literature findings obtained in different experimental cell models indicating that high $\beta$-catenin expression can trigger NF- $\kappa \mathrm{B}$-dependent upregulation of hepatocyts inducible nitric oxide synthase (iNOS) that modulating survivin levels mediated via PI3k/Akt pathway $[21,22]$. Thus, it is obvious that the iNOS/survivin-pathway can bestow cytoprotective effect and confer radioresistance in HCC Mahlavu cells (Figure 4(a)). In parallel with these data, the effect of NO production on COX-2 overexpression and the latter is able to stabilize survivin in cnacer cells other than HCC has been documented [23,24]. Therefore, high iNOS/survivinpathway associated with HCC Mahlavu cells may also be 
linked to NO-mediated overexpression of COX-2 that confer the stabilization of survivin. This possibility can not be ruled out. Collectively, our data reveal that iNOS/ survivin-targeting approach may be a potential strategy for overcoming radioresistance in HCC cells besides Bcl-2 [25].

In line with the finding that radioresistant HCC Mahlavu cells overexpressed Bcl-2 and survivin, these cells can also uniquely and highly boost their production of glucose-regulated protein 78 (GRP-78) (Figure 4(d)). GRP-78, a stress-inducible endoplasmic reticulum (ER) chaperone, that is associated with the unfolded protein response (UPR), a defense cellular mechanism activated during such stress condition [26-28]. Due to its pivotal role in UPR, GRP78 is overexpressed in many cancers including HCC as depicted in this study; it is now demonstrated to play a significant role in cancer cells survival through supporting of drug and radioresistance as well as metastatic dissemination [26]. For these reasonings, we thus set out to explore the mechanistic pathways that may be involved in conferring radioresistance in HCC Mahlavu cells. As shown in Figure 4(b), using DAF-DA and Fluo 4 as the probes for confocal microscopic detection of intracellular nitric oxide (NO) and cytosolic calcium $\left(\mathrm{cCa}^{2+}\right)$, respectively, we unveiled that radioresistant Mahlavu cells, genetically endowed with higher iNOS, were shown to produce NO plethorically in a mild sustained fashion. Consequently, NO-mediated perturbation of $\mathrm{cCa}^{2+}$ homeostasis ensured. The overflow of $\mathrm{cCa}^{2+}$, probably released from ER, caused ER stress response that eventually activated the cleavage of ER's ATF- $6 \alpha$ $(90 \mathrm{kDa})$ transcriptional factor to it's ATF- $6 \alpha(50 \mathrm{kDa})$ subunit that translocated to mitochondria to release GRP-78 (Figure 5). Interestingly, siRNA silencing of either survivin or GRP78, the cleavage of ATF- $6 \alpha$ (90 $\mathrm{kDa}$ ) was shown to be completely blocked with concomitant downregulation of GRP78 expression implying the cross-talk between cellular survivin and GRP-78. The interaction of survivin and GRP-78 has not previously been reported elsewhere. Even more importantly, both siRNA silencing of survivin and GFP-78 caused drastic downregulation $\beta$-catenin implying that they are twosides of the same coin. Severely downregulated $\beta$-catenin by siRNA silencing of survivin and GRP-78 can be used to interpret the reasoning of why knocking down GRP-78 causes resistance to curcumin treatment through the suppression of caspase- 3 and caspase- 8 expression levels. This is probably due to the fact that cucumin suppresses Bcl-2 expression [16], but severely retaining survivin, a radioresistant effector, even with GRP-78 knockdown of Mahlavu cells, that still can maintain its radioresistance acquisition as previously described by us elsewhere [29]. Taken together, our data clearly demonstrated that con- stitutively overexpressed GRP-78 is mediated through $\beta$-catenin/NF- $\kappa$ B/iNOS pathway which causes ER stress response by activating ATF-6 $\alpha(90 \mathrm{kDa})$ transcription factor breakdown to its clevaged ATF- $6 \alpha(50 \mathrm{kDa})$ subunit which translocates to mitochondria to release GRP78. With the elucidation of this mechanistic pathway, it is possible to employ (-)-Epigallocatechin gallate (EGEG) to overcome Soratenib's resistance in HCC cells since we have previously demonstrated that GRP-78 plays a pivotal role in conferring therapeutic resistance to this multi-kinase inhibitor drug [7,30]. Thus, the recent discovery of an alternative pathway indicating that Sorafenib-induced oxidative-nitrosative stress can be served as a radiosensitizing agent to improve the efficacy of radiotherapy. This possibility is currently under investigation in our laboratories.

\section{Acknowledgements}

This study was supported by grant from Chi-Mei Medical Research Foundation (98CM-TMU-01-01). The editorial assistance of Miss Wei-Ching Lo is greatly appreciated.

\section{REFERENCES}

[1] G. M. T. Kuo and N. Savaraj, "Roles of Reactive Oxygen Species in Hepatocarcinogenesis and Drug Resistance Gene Expression in Liver Cancers," Molecular Carcinogenesis, Vol. 45, No. 9, 2006, pp. 701-709. doi: $10.1002 / \mathrm{mc} .20240$

[2] D. M. Parkin, F. Bray, J. Ferlay and P. Pisani, "Global Cancer Statistics, 2002," CA: A Cancer Journal for Clinicians, Vol. 55, No. 2, 2005, pp. 74-108. doi:55/2/74

[3] T. Zhang, H. Liu, C. Zhu, K. Briggs, Y. Kang, J. A. Fleming and S. A. Curley, "Silencing Thioredoxin Induces Liver Cancer Cell Senescence under Hypoxia," Hepatology Research, Vol. 42, No. 7, 2012, pp. 706-713. doi:10.1111/j.1872-034X.2012.00973.x

[4] C. C. Lin and M. C. Yin, "B Vitamins Deficiency and Decreased Anti-Oxidative State in Patients with Liver Cancer," European Journal of Nutrition, Vol. 46, No. 5, 2007, pp. 293-299. doi:10.1007/s00394-007-0665-8

[5] B. I. Carr, "Hepatocellular Carcinoma: Current Management and Future Trends," Gastroenterology, Vol. 127, No. 5, 2004, pp. S218-S224. doi:10.1053/i.gastro.2004.09.036

[6] M. Garcia-Barros, F. Paris, C. Cordon-Cardo, D. Lyden, S. Rafii, A. Haimovitz-Friedman, Z. Fuks and R. Kolesnick, "Tumor Response to Radiotherapy Regulated by Endothelial Cell Apoptosis," Science, Vol. 300, No. 5622, 2003, pp. 1155-1159. doi:10.1126/science.1082504

[7] J. F. Chiou, C. J. Tai, M. T. Huang, P. L. Wei, Y. H. Wang, J. An, C. H. Wu, T. Z. Liu and Y. J. Chang, "Glucose-Regulated Protein 78 Is a Novel Contributor to Acquisition of Resistance to Sorafenib in Hepatocellular Carcinoma," Annals of Surgical Oncology, Vol. 17, No. 2, 
2010, pp. 603-612. doi:10.1245/s10434-009-0718-8

[8] C. J. Tai, H. Chin-Sheng, L. J. Kuo, P. L. Wei, H. H. Lu, H. A. Chen, T. Z. Liu, J. J. Liu, D. Z. Liu, Y. S. Ho, C. H. $\mathrm{Wu}$ and Y. J. Chang, "Survivin-Mediated Cancer Cell Migration through Grp78 and Epithelial-Mesenchymal Transition (Emt) Marker Expression in Mahlavu Cells," Annals of Surgical Oncology, Vol. 19, No. 1, 2012, pp. 336-343. doi:10.1245/s10434-011-1692-5

[9] R. J. Bold, P. M. Termuhlen and D. J. McConkey, "Apoptosis, Cancer and Cancer Therapy," Surgical Oncology, Vol. 6, No. 3, 1997, pp. 133-142. doi:S0960-7404(97)00015-7

[10] L. T. Condon, J. N. Ashman, S. R. Ell, N. D. Stafford, J. Greenman and L. Cawkwell, "Overexpression of Bcl-2 in Squamous Cell Carcinoma of the Larynx: A Marker of Radioresistance," International Journal of Cancer, Vol. 100, No. 4, 2002, pp. 472-475. doi:10.1002/ijc.10503

[11] O. Gallo, V. Boddi, A. Calzolari, L. Simonetti, M. Trovati and S. Bianchi, "Bcl-2 Protein Expression Correlates with Recurrence and Survival in Early Stage Head and Neck Cancer Treated by Radiotherapy," Clinical Cancer Research, Vol. 2, No. 2, 1996, pp. 261-267.

[12] P. Nix, L. Cawkwell, H. Patmore, J. Greenman and N. Stafford, "Bcl-2 Expression Predicts Radiotherapy Failure in Laryngeal Cancer," British Journal of Cancer, Vol. 92, No. 12, 2005, pp. 2185-2189. doi:10.1038/sj.bjc.6602647

[13] E. M. Rosen, S. Fan, S. Rockwell and I. D. Goldberg, "The Molecular and Cellular Basis of Radiosensitivity: Implications for Understanding How Normal Tissues and Tumors Respond to Therapeutic Radiation," Cancer Investigation, Vol. 17, No. 1, 1999, pp. 56-72.

[14] A. Strasser, A. W. Harris and S. Cory, "Bcl-2 Transgene Inhibits T Cell Death and Perturbs Thymic Self-Censorship," Cell, Vol. 67, No. 5, 1991, pp. 889-899. doi:0092-8674(91)90362-3

[15] A. Kardosh, N. Soriano, P. Pyrko, Y. T. Liu, M. Jabbour, F. M. Hofman and A. H. Schonthal, "Reduced Survivin Expression and Tumor Cell Survival during Chronic Hypoxia and Further Cytotoxic Enhancement by the Cyclooxygenase-2 Inhibitor Celecoxib," Journal of Biomedical Science, Vol. 14, No. 5, 2007, pp. 647-662. doi:10.1007/s11373-007-9173-3

[16] S. K. Sandur, A. Deorukhkar, M. K. Pandey, A. M. Pabon, S. Shentu, S. Guha, B. B. Aggarwal and S. Krishnan, "Curcumin Modulates the Radiosensitivity of Colorectal Cancer Cells by Suppressing Constitutive and Inducible Nf-Kappab Activity," International Journal of Radiation Oncology*Biology*Physics, Vol. 75, No. 2, 2009, pp. 534542. doi:10.1016/j.ijrobp.2009.06.034

[17] J. F. Liu, G. G. Jamieson, P. A. Drew, G. J. Zhu, S. W. Zhang, T. N. Zhu, B. E. Shan and Q. Z. Wang, "Aspirin Induces Apoptosis in Oesophageal Cancer Cells by Inhibiting the Pathway of Nf-Kappab Downstream Regulation of Cyclooxygenase-2," ANZ Journal of Surgery, Vol. 75, No. 11, 2005, pp. 1011-1016. doi:10.1111/j.1445-2197.2005.03596.x

[18] M. Pennati, M. Folini and N. Zaffaroni, "Targeting Survivin in Cancer Therapy: Fulfilled Promises and Open
Questions," Carcinogenesis, Vol. 28, No. 6, 2007, pp. 1133-1139. doi:10.1093/carcin/bgm047

[19] K. Asanum, R. Moriai and T. Yajima, "Survivin as a Radioresistant Factor in Pancreatic Cancer," Japanese Journal of Cancer Research, Vol. 91, No. 11, 2000, pp. 1204-1209.

[20] N. K. Sah, A. Munshi, M. Hobbs, B. Z. Carter, M. Andreeff and R. E. Meyn, "Effect of Downregulation of Survivin Expression on Radiosensitivity of Human Epidermoid Carcinoma Cells," International Journal of Radiation Oncology*Biology*Physics, Vol. 66, No. 3, 2006, pp. 852-859. doi:10.1016/j.ijrobp.2006.06.049

[21] A. Bandino, A. Compagnone, V. Bravoco, C. Cravanzola, A. Lomartire, C. Rossetto, E. Novo, S. Cannito, L. Valfre di Bonzo, E. Zamara, R. Autelli, M. Parola and S. Colombatto, "Beta-Catenin Triggers Nuclear Factor Kappab-Dependent Up-Regulation of Hepatocyte Inducible Nitric Oxide Synthase," The International Journal of Biochemistry \& Cell Biology, Vol. 40, No. 9, 2008, pp 1861-1871. doi:10.1016/j.biocel.2008.01.029

[22] V. Fetz, C. Bier, N. Habtemichael, R. Schuon, A. Schweitzer, M. Kunkel, K. Engels, A. F. Kovacs, S. Schneider, W. Mann, R. H. Stauber and S. K. Knauer, "Inducible No Synthase Confers Chemoresistance in Head and Neck Cancer by Modulating Survivin," International Journal of Cancer, Vol. 124, No. 9, 2009, pp. 2033-2041. doi:10.1002/ijc.24182

[23] K. Krysan, F. H. Merchant, L. Zhu, M. Dohadwala, J. Luo, Y. Lin, N. Heuze-Vourc'h, M. Pold, D. Seligson, D. Chia, L. Goodglick, H. Wang, R. Strieter, S. Sharma and S. Dubinett, "Cox-2-Dependent Stabilization of Survivin in Non-Small Cell Lung Cancer," The FASEB Journal, Vol. 18, No. 1, 2004, pp. 206-208. doi:10.1096/fj.03-0369fje

[24] S. W. Park, S. G. Lee, S. H. Song, D. S. Heo, B. J. Park, D. W. Lee, K. H. Kim and M. W. Sung, "The Effect of Nitric Oxide on Cyclooxygenase-2 (Cox-2) Overexpression in Head and Neck Cancer Cell Lines," International Journal of Cancer, Vol. 107, No. 5, 2003, pp. 729-738. doi:10.1002/ijc.11498

[25] H. Yamamoto, C. Y. Ngan and M. Monden, "Cancer Cells Survive with Survivin," Cancer Science, Vol. 99, No. 9, 2008, pp. 1709-1714. doi:10.1111/j.1349-7006.2008.00870.x

[26] M. V. Backer, J. M. Backer and P. Chinnaiyan, "Targeting the Unfolded Protein Response in Cancer Therapy," Methods in Enzymology, Vol. 491, 2011, pp. 37-56. doi:10.1016/B978-0-12-385928-0.00003-1

[27] T. Y. Lin, J. T. Chang, H. M. Wang, S. H. Chan, C. C. Chiu, C. Y. Lin, K. H. Fan, C. T. Liao, I. H. Chen, T. Z. Liu, H. F. Li and A. J. Cheng, "Proteomics of the Radioresistant Phenotype in Head-and-Neck Cancer: Gp96 as a Novel Prediction Marker and Sensitizing Target for Radiotherapy," International Journal of Radiation Oncology*Biology*Physics, Vol. 78, No. 1, 2010, pp. 246-256. doi:10.1016/j.ijrobp.2010.03.002

[28] M. J. Wu, C. I. Jan, Y. G. Tsay, Y. H. Yu, C. Y. Huang, S. C. Lin, C. J. Liu, Y. S. Chen, J. F. Lo and C. C. Yu, 
"Elimination of Head and Neck Cancer Initiating Cells through Targeting Glucose Regulated Protein78 Signaling," Molecular Cancer, Vol. 9, 2010, p. 283. doi:10.1186/1476-4598-9-283

[29] Y. J. Chang, C. J. Tai, L. J. Kuo, P. L. Wei, H. H. Liang, T. Z. Liu, W. Wang, Y. S. Ho, C. H. Wu and M. T. Huang, "Glucose-Regulated Protein 78 (Grp78) Mediated the Efficacy to Curcumin Treatment on Hepatocellular Carcinoma," Annals of Surgical Oncology, Vol. 18, No. 8,
2011, pp. 2395-2403. doi:10.1245/s10434-011-1597-3

[30] S. P. Ermakova, B. S. Kang, B. Y. Choi, H. S. Choi, T. F. Schuster, W. Y. Ma, A. M. Bode and Z. Dong, "(-)-Epigallocatechin Gallate Overcomes Resistance to EtoposideInduced Cell Death by Targeting the Molecular Chaperone Glucose-Regulated Protein 78," Cancer Research, Vol. 66, No. 18, 2006, pp. 9260-9269. doi:10.1158/0008-5472.CAN-06-1586 